\title{
Critical illness neuropathy
}

\section{J. Vijayan, M. Alexander}

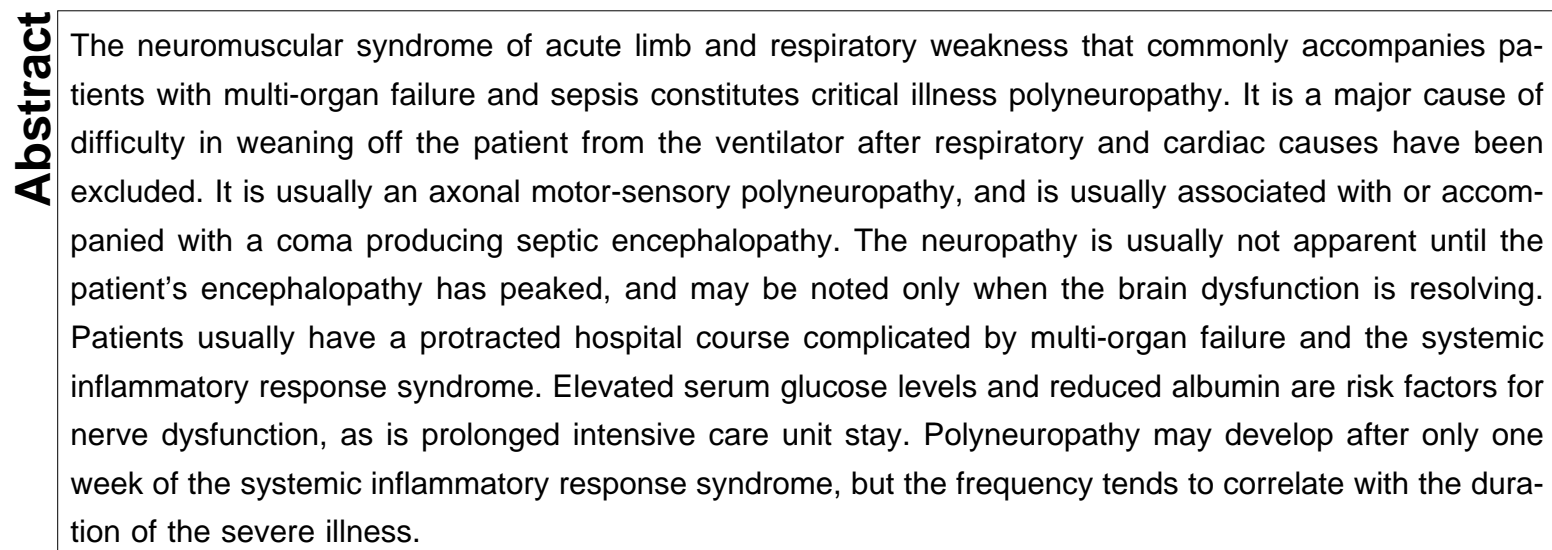

Key Words: Critical Care, SIRS, Axonal Polyneuropathy

The neuromuscular syndrome of acute limb and respiratory weakness that commonly accompanies patients with multi-organ failure and sepsis constitutes critical illness polyneuropathy. It is a major cause of difficulty in weaning off the patient from the ventilator after respiratory and cardiac causes have been excluded. It is usually an axonal motor-sensory polyneuropathy, and is usually associated with or accompanied with a coma producing septic encephalopathy.

The neuropathy is usually not apparent until the patient's encephalopathy has peaked, and may be noted only when the brain dysfunction is resolving. The patient often cannot be weaned from the ventilator because of the phrenic nerve involvement. Distal muscle weak-

\section{From:}

Department of Neurological Sciences, Christian Medical College Hospital, Vellore, India

Correspondence:

Dr. Mathew Alexander,

Professor of Neurology, Dept of Neurological Sciences, Christian Medical

College Hospital, Vellore - 632 004, India. E-mail: mathewalex@cmcvellore.ac.in ness and loss of deep tendon reflexes are usually found. The cranial nerve musculature is relatively spared. Pain and paraesthesias are usually absent in patients with critical illness polyneuropathy.

Patients usually have a protracted hospital course complicated by multi-organ failure and the systemic inflammatory response syndrome (SIRS). Corticosteroids and neuromuscular blockade agents are not recognized as risk factors in its causation; however the potential role of antibiotics remains unresolved. Elevated serum glucose levels and reduced serum albumin are risk factors for nerve dysfunction, as is prolonged intensive care unit stay. Polyneuropathy may develop as early as one week after the onset of SIRS, but the incidence tends to correlate with the duration of the severe illness. Therefore, patients presenting with significant neuromuscular weakness developing within hours to days of the illness are unlikely to have critical illness polyneuropathy. On the other hand, they are more likely to have neuropathy of another etiology, critical illness myopathy, or a neuromus- 
cular junction disorder. Likewise, critical illness polyneuropathy does not develop after the patient has been discharged from the hospital. Toxic or immune mediated neuropathies are more likely at that stage.

The pathogenesis of critical illness polyneuropathy is not known. A prospective study by Witt and colleagues showed that the development of critical illness neuropathy was associated with hyperglycaemia and inversely related to hypoalbuminaemia. This is consistent with an underlying systemic inflammatory response syndrome. Bolton has proposed a disturbance in the microvascular function in peripheral nerves to be responsible for the neuropathy. The primary axonal damage may be due to the involvement of axonal transport system, which is known to be energy dependent; this fact may explain the predominantly distal nerve segment involvement. Moreover it is known that the blood nerve barrier, in contrast to the blood brain barrier, shows increased permeability to histamine and serotonin. Several mediators of the septic syndrome are known to have histamine-like action. Circulating toxins could potentially gain access to the endoneural space and directly damage the axon. ${ }^{[1,2]}$ Additional theories include: (1) axonal degeneration due to glucose-induced phosphate depletion from parenteral nutrition with subsequent depletion of high energy phosphate compounds; (2) damage of neural microvasculature due to oxidative effects of parenterally administered lipids; (3) impaired transport of axonal proteins and (4) toxic effects from the release of proinflammatory cytokines.

Critical illness polyneuropathy must also be differentiated from other acute polyneuropathies, including Guillian Barre syndrome, neuropathies of metabolic origin (e.g., vitamin deficiency, hypophosphatemia, porphyria), toxic effects of antibiotics (metranidazole, aminoglycosides or penicillin) and the remote effects of cancer (paraneoplastic neuropathy). It should also be differentiated from neuromuscular blockade and from critical illness myopathy. Critical illness myopathy should be especially looked into as the clinical setting is almost similar to that of critical illness polyneuropathy. This was initially described in patients with asthma, who had received high dose of parenteral steroids and in those with sepsis who had received nondepolarizing neuromuscular blocking agents. A significant number of patients with organ transplantation also go on to develop critical ill- ness myopathy. Muscle histopathology reveals myopathy with loss of thick filaments in almost $80 \%$, mild myopathic changes in $14 \%$ and atrophy of type I and II fibers in a small number. Selective loss of thick filaments is particularly associated with the use of intravenous corticosteroids.

Electrophysiologic findings are those of a pure axonal degeneration, affecting motor than sensory fibers. Conduction velocities and distal latencies are relatively intact, but there is a reduction in the compound muscle and the sensory nerve action potentials. Needle EMG reveals fibrillation potentials and positive sharp waves indicating active denervation. Even in the more advanced stages of critical illness polyneuropathy, conduction velocities and distal latencies remain relatively normal, emphasizing the purely axonal nature of the neuropathy ${ }^{[3]}$ In the acute setting, if sensory response is normal, a diagnosis of critical illness "motor" polyneuropathy should not be made without excluding myopathy.

Laboratory investigations are nonspecific, and CSF analysis reveals normal to mildly elevated proteins. Nerve biopsy reveals axonal degeneration without evidence of demyelination or inflammation. Muscle biopsy shows scattered atrophic fibers in cases with recent denervation and grouped muscle fiber atrophy in longstanding cases. Comprehensive examination of the entire nervous system at autopsy, plus nerve and muscle biopsy has revealed that that there is a primary axonal degeneration of the motor and sensory fibers, particularly involving distal nerve fibers. There is a resulting denervation atrophy of muscle; histopathological examination during the acute phase reveals scattered, angulated fibers and later shows grouped atrophy. Neither the nerve nor the muscle shows any inflammatory changes. Aside from chromatolysis of the anterior horn cells secondary to the peripheral axonal injury, the central nervous system is spared. Comprehensive studies of the critically ill patients by Latronica ${ }^{[4]}$ and associates revealed that some patients have abnormal electrophysiology of the nerve and muscle, but normal nerve and muscle biopsies, suggesting that functional changes precede structural alterations.

Treatment is supportive, initially consisting of aggressive pulmonary hygiene and prevention of secondary 
complications of immobility such as skin breakdown, deep venous thrombosis and superimposed compressive neuropathies. Hypoalbuminemia and hyperglycaemia should be corrected. Some patients, particularly those with gram-negative sepsis, may benefit from intravenous immunoglobulins; yet widespread acceptance of this treatment awaits further supportive data. ${ }^{[5,6]}$ Long term treatment may consist of rehabilitation, assistive devices, and medication for neuropathic pain, if present. Recovery from critical illness polyneuropathy takes months to years and is often incomplete. Clinical deficits are present in $59 \%$ of survivors and electrophysiologic abnormalities in $95 \%$ of survivors followed upto 5 years. ${ }^{[7]}$

\section{References}

1. Witt NJ, Zochodne DW, Bolton CF, Grand'Maison F, Wells G, Young GB, et al. Peripheral nerve function in sepsis and Mulitorgan failure. Chest 1991;99:176-84.

2. Bolton CF, Gilbert JJ, Hahn AF, Sibbald WJ. Polyneuropathy in critically ill patients. J Neurol Neurosurg Psychiatr 1984;47: 1223-31.

3. Ziko UA, Zipco HT, Bolton CF. Clinical and electrophysiological findings in critical illness polyneuropathy. J Neurol Sci 1998;158:186-93.

4. Latronico N, Fenzi F, Recupero D, Guarneri B, Tomelleri G, Tonin $P$, et al. Critical illness myopathy and neuropathy. Lancet 1996;247:1579-82.

5. Wijdicks EF, Fulgham JR. Failure of high dose intravenous immunoglobulins to alter the clinical course of critical illness polyneuropathy. Muscle Nerve 1994;17:1494-5.

6. Mohr M, Englisch L, Roth A, Burchardi H, Zielmann S. Effects of early treatment with immunoglobulins on critical illness polyneuropathy following multiple organ failure and gram negative sepsis. Intensive Care Med 1997;23:1144-9.

7. Fletcher SN, Kennedy DD, Ghosh IR, Misra VP, Kiff K, Coakley $\mathrm{JH}$, et al. Persistent neuromuscular and neurophysiologic abnormalities in long term survivors of prolonged critical illness. Crit Care Med 2003;31:1012-6. 